\title{
Victims of rape show increased cortisol responses to trauma reminders: A study in individuals with war- and torture-related PTSD
}

\author{
Hannah Gola ${ }^{a}$, Harald Engler ${ }^{b}$, Maggie Schauer ${ }^{a}$, Hannah Adenauer ${ }^{a}$, \\ Carsten Riether ${ }^{c}$, Stephan Kolassa ${ }^{\mathrm{d}}$, Thomas Elbert ${ }^{\mathrm{a}}$, Iris-Tatjana Kolassa ${ }^{\mathrm{a}, \mathrm{e}, \mathrm{f}, *}$ \\ ${ }^{a}$ Clinical Psychology \& Neuropsychology, University of Konstanz, Germany \\ ${ }^{\mathrm{b}}$ Institute of Medical Psychology and Behavioral Immunobiology, University Hospital Essen, University of Duisburg-Essen, Germany \\ 'Laboratory of Psychology and Behavioral Immunobiology, Institute for Behavioral Sciences, ETH Zurich, Switzerland \\ ${ }^{\mathrm{d}}$ Research \& Innovation, SAF Simulation, Analysis \& Forecasting AG, Tägerwilen, Switzerland \\ e Zukunftskolleg, University of Konstanz, Germany \\ ${ }^{f}$ Clinical \& Biological Psychology, University of Ulm, Germany
}

\section{KEYWORDS}

Posttraumatic stress

disorder;

Cortisol;

Rape;

Trauma type;

Tonic immobility;

Dissociation

\begin{abstract}
Summary Studies investigating cortisol responses to trauma-related stressors in patients with posttraumatic stress disorder (PTSD) have yielded inconsistent results, demonstrating that cortisol responses were enhanced or unaffected when confronted with trauma reminders. This study investigated the effect of the type of trauma experienced on both salivary and plasma cortisol responses during confrontation with trauma-related material. Participants were 30 survivors of war and torture, with and without rape among the traumatic events experienced. Participants of both groups (raped vs. non-raped) fulfilled DSM-IV criteria of PTSD. Plasma and salivary cortisol levels were measured at three time points during a standardized clinical interview: once before and twice after assessing individual traumatic experiences. Results show that groups did not differ in basal plasma and salivary cortisol levels. However, differential salivary cortisol responses were observed in PTSD patients who had been raped compared to those who had not been raped $(p<.05)$ but had experienced an equal number of traumatic events and showed equally high PTSD symptom severity. Whereas salivary cortisol levels decreased in the course of the interview for the group with no past experience of rape $(p<.05)$, those PTSD patients who had been raped showed a significant cortisol increase when reminded of their traumatic events $(p<.001)$. This effect was not found in plasma cortisol. Our results indicate that the type of traumatic stress experienced contributes to cortisol
\end{abstract}

\footnotetext{
* Corresponding author at: University of Ulm, Department of Clinical \& Biological Psychology, Albert-Einstein-Allee 47, 89069 Ulm, Germany. Tel.: +497315026590; fax: +497315026599.

E-mail address: Iris. Kolassa@uni-ulm.de (I.-T. Kolassa).
} 
responses during the confrontation with trauma-related material. We hypothesize, that the nearness of the perpetrator during the traumatic event might shape later psychophysiological responding to trauma reminders.

\section{Introduction}

The experience of traumatic events such as life-threatening accidents, combat exposure, or rape poses a risk for the development of posttraumatic stress disorder (PTSD), characterized by intrusive recollections of the trauma, persistent avoidance of trauma reminders, emotional numbing and hyperarousal (American Psychiatric Association, 1994). An abundance of studies have documented long-term alterations in the functioning of the hypothalamus-pituitary-adrenal (HPA) axis in PTSD patients. These are characterized mostly by lower (Yehuda et al., 1995) but also by normal (Tucker et al., 2004) or higher (Lindauer et al., 2006) basal cortisol levels, with a recent meta-analysis concluding that low cortisol levels in PTSD are only found under certain conditions, such as in studies with unexposed control subjects, in studies on abuse, and in studies including female subjects (Meewisse et al., 2007). Furthermore altered diurnal patterns of cortisol release have been reported (Wessa et al., 2006). In addition to the investigation of basal alterations in PTSD patients, several challenge paradigms (e.g., cognitive or pharmacological) have been developed to examine alterations in the responsivity of the hormonal stress system in PTSD patients (for a review see de Kloet et al., 2006). In this context, earlier studies also investigated cortisol reactivity in PTSD patients in response to trauma reminders, yielding inconsistent results. Whereas a study by Elzinga et al. (2003) reported a pronounced cortisol response in sexually abused women when confronted with personal trauma scripts, other studies examining cortisol reactivity after assessing individual traumatic experiences in male torture victims (Kolassa et al., 2007) or in response to combat noise in male Vietnam veterans (Liberzon et al., 1999) found no such response.

Based on current theoretical models of PTSD (Lang, 1979; Foa and Kozak, 1986; Schauer et al., 2011), studies investigating physiological responses to trauma-related cues generally assume that the physiological reactivity in response to trauma reminders observed in the laboratory should recapitulate the physiological reactivity that occurred during the trauma (Keane et al., 1985; Pole, 2007). Whereas earlier studies proposed heightened autonomic responses of PTSD patients to trauma reminders in general (Orr and Roth, 2000), more recent investigations suggest that PTSD patients who responded to trauma with a drop in arousal, such as immobility or fainting - a response set which we refer to as "dissociative shut-down" (Schauer and Elbert, 2010), show reduced autonomic psychophysiological activity and reactivity when confronted with trauma reminders (Griffin et al., 1997; Lanius et al., 2002; Pole, 2007). If trauma reminders replay aspects of the peritraumatic response mode, different physiological response patterns should also be seen during exposure treatment depending on whether the original traumatic situation had predominantly activated the sympathetic "flight-fight" or had likely driven the defensive system into immobility and "shut-down" (Schauer and Elbert, 2010). This assumption has been confirmed by neuroimaging studies, showing differential neural activation patterns for the two response types in response to trauma-related stimulation (Lanius et al., 2006, 2010).

Interestingly, studies suggest that these different peritraumatic response modes of trauma victims tend to vary with the type of trauma experienced. For example, Kaysen et al. (2005) investigated women's peritraumatic responses to different forms of crimes and found that approximately one third of women reported active physical resistance (e.g., biting, cursing, kicking), whereas a large proportion of women, especially victims of rape, reported non-active behavioral responses, i.e., "shut-down" responses. In victims of sexual assault, the failure to escape or fight an assailant is typically followed by "rape-induced paralysis" (Burgess and Holmstrom, 1976), or tonic immobility (TI, Suarez and Gallup, 1979; Marx et al., 2008). During such an episode of rape-induced paralysis or TI, sexual assault victims find themselves unable to move, resist or incapable of calling out for help, while remaining conscious - eventually resulting in many cases in flaccid and unresponsive immobility or even fainting, mediated by vaso-vagal syncope (Schauer and Elbert, 2010). Empirical studies show that a substantial number (37-52\%) of survivors experiences some form of immobility during their sexual assault (Galliano et al., 1993; Heidt et al., 2005; Fuse et al., 2007). The probability of experiencing immobility is even more augmented when the sexual assault involves the attempt of or the act of vaginal coitus (Heidt et al., 2005).

Here we investigate whether the type of trauma (rape vs. other trauma types) is associated with differential stress responses when confronted with trauma-related material, using cortisol as a physiological stress marker. For that purpose, both salivary and total plasma cortisol levels of PTSD patients with severe traumata, including or not including rape, were measured at three time points during a detailed and standardized clinical interview: once before and twice after assessing individual traumatic experiences such as rape, war and torture with standardized checklists. Furthermore, basal cortisol levels of PTSD patients were compared with those in a third healthy control group.

\section{Materials and methods}

\subsection{Participants}

Cortisol reactivity was assessed in 30 individuals with current PTSD according to DSM-IV (American Psychiatric Association, 1994), recruited from the Psychotrauma Research and Outpatient Clinic for Refugees, University of Konstanz. All PTSD patients were refugees ( 4 from Africa, 5 Balkan, 21 Middle East and Afghanistan) with multiple highly stressful war and torture experiences, including or not including rape. On average, patients had lived in Germany for 6.4 years $(S D=4.9)$. 
To investigate the influence of trauma type on cortisol reactivity, patients were divided into two groups: raped $(n=10 ; 6$ female, 4 male; mean age $=34.0$ years, $S D=10.2)$ vs. non-raped $(n=20 ; 8$ female, 12 male, mean age $=33.7$ years, $S D=8.7$ ). Rape was defined as any traumatic stressor involving unwanted vaginal or anal penetration obtained by the use of force or threat of force. Ten individuals $(100 \%)$ out of the raped group and 14 patients (70\%) of the non-raped group met the DSM-IV criteria for a current major depressive episode. $40 \%$ of the raped group and $35 \%$ of the non-raped group reported current intake of hypnotic, anxiolytic, antidepressant or neuroleptic medication. With the exception of one woman within the raped group, all of the patients were free of oral contraceptives. Three individuals (30\%) out of the raped group and 8 patients (40\%) of the non-raped group were smokers. Subjects were excluded, if they reported intake of glucocorticoids, had a significant acute or chronic medical illness, or met criteria for additional mental disorders according to DSM-IV other than stress-related affective or anxiety disorders.

To compare basal cortisol levels of the PTSD group with a non-PTSD group, we further examined a third healthy control group ( $n=28 ; 17$ female, 11 male; mean age $=26.9$ years, $S D=7.0$ ). This group was recruited through advertisement and was parallelized to the patient group with regard to region of origin (5 Africa, 11 Balkan, 12 Middle East and Afghanistan). With the exception of one subject, all controls reported at least one $(M=3.3, S D=2.2)$ traumatic event type on the event checklist of the Clinician-Administered PTSD Scale (CAPS, Blake et al., 1995). Moreover, 11 control subjects reported items $(M=2.2, S D=4.8)$ on the vivo checklist of war, detention and torture events (Schauer et al., 2011). Five control subjects (17.86\%) were smokers. Except for four women reporting the intake of oral contraceptives, all control subjects were free of medication.

\subsection{Procedure}

Plasma and salivary cortisol levels of PTSD patients were measured at three time points during a detailed and standardized clinical interview, starting at 10:00 a.m., once before and twice after assessing individual rape, war and torture experiences (see Fig. 1). The clinical interviews were conducted by experienced clinical psychologists with the help of trained translators. All procedures were approved by the Ethics Committee of the University of Konstanz and were carried out in accordance with the Declaration of Helsinki 2008.
Upon arrival at the outpatient clinic, procedures were explained to the participants and written informed consent was obtained. Subsequently, the first blood sample $\left(t_{1}\right)$ for basal plasma cortisol measurement was drawn into an EDTAtreated tube (BD Vacutainer, Franklin Lakes, NY, USA). In addition, a saliva sample was obtained with a commercially available collection device (Salivette, Sarstedt, Nümbrecht, Germany). Afterwards, sociodemographic information as well as medical information was acquired. Somatic symptoms were assessed using a shortened version of Screening for Somatoform Symptoms-7 (SOMS-7, Rief and Hiller, 2003). During the second part of the interview, participants were interviewed in a standardized manner about their individual child and adulthood traumatic experiences using the event checklist of the CAPS (Blake et al., 1995) and the vivo checklist of war, detention and torture events (Schauer et al., 2011), which assesses common traumatic experiences in conflict regions and during torture. This phase of the interview took on average $50 \mathrm{~min}(S D=16 \mathrm{~min})$. Immediately afterwards, blood and saliva samples were collected for the second time $\left(t_{2}\right)$. In the third part of the interview, PTSD symptom frequency and severity were assessed with the CAPS (Blake et al., 1995) and blood and saliva samples were collected for the third time $\left(t_{3}\right)$. Finally, the Mini International Neuropsychiatric Interview (M.I.N.I., Sheehan et al., 1998) was applied to screen for potential comorbid mental disorders. In addition, the severity of depressive symptoms was assessed with the Hamilton Depression Rating Scale (HAM-D, Hamilton, 1960). One week after this standardized clinical interview the participants were invited to the lab for a second time. Again, blood and saliva samples were collected at 10:00 a.m. $\left(t_{4}\right)$, to test for the stability of basal $\left(t_{1}\right)$ cortisol secretion. Subsequent to these procedures, all patients were offered a trauma-focused psychotherapy at our institution.

Non-PTSD control subjects underwent the same standardized clinical interview as PTSD patients, but blood and saliva was taken only at the beginning of the interview $\left(t_{1}\right)$ as well as one week afterwards $\left(t_{4}\right)$.

\subsection{Cortisol analysis}

Immediately after blood collection, plasma was separated $\left(3000 \times \mathrm{g}, 10 \mathrm{~min}, 4^{\circ} \mathrm{C}\right)$ and stored at $-28^{\circ} \mathrm{C}$ until analysis. Saliva collection devices were centrifuged $(1400 \times \mathrm{g}, 3 \mathrm{~min}$, $4^{\circ} \mathrm{C}$ ) and the recovered saliva was stored at $-28^{\circ} \mathrm{C}$ until assayed.

Salivary cortisol concentrations were measured by a beadbased competitive binding assay. Undiluted saliva and corti-

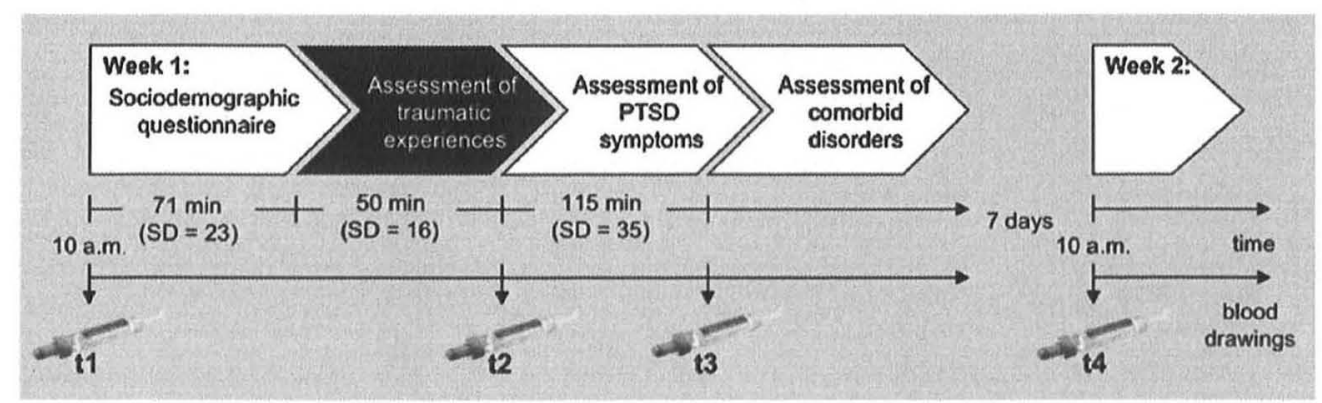

Figure 1 Schematic description of the procedures. 
sol standards were incubated overnight in 96-well microtiter plates with cortisol-BSA-conjugated polystyrene beads and fluorescein isothiocyanate (FITC)-conjugated rabbit anti-cortisol antibody (HTB192, Chromaprobe, Maryland Heights, MO, USA) at room temperature. After incubation, beads were washed and resuspended in phosphate-buffered saline containing $0.05 \%$ Tween-20, and analyzed on flow cytometer equipped with high-throughput sampler (LSR II, BD Immunocytometry Systems, San Jose, CA, USA). Antibody crossreactivity with other relevant steroids was $4.0 \%$ (testosterone) and $0.9 \%$ (progesterone), respectively. Intra- and interassay variance were $5.4 \%$ and $10.7 \%$, respectively.

Total plasma cortisol concentrations were measured using a commercial enzyme-linked immunosorbent assay (Cortisol ELISA, IBL International, Hamburg, Germany) according to the manufacturer's instructions. Intra- and interassay variance were $5.6 \%$ and $6.9 \%$, respectively.

\subsection{Statistical analyses}

Data analysis was performed using SPSS 17.0. Group differences in demographic and clinical variables were analyzed using $t$-tests for independent samples. Salivary and plasma cortisol reactivity were analyzed using mixed model ANOVAs with trauma type (raped vs. non-raped) or trauma type and gender or current depression as fixed effect and time $\left(t_{1}, t_{2}\right.$, and $t_{3}$ ) as repeated measure factor. Group differences in basal salivary and plasma cortisol levels were analyzed using mixed model ANOVAs with diagnosis (PTSD vs. controls) as fixed effect and time $\left(t_{1}\right.$ and $\left.t_{4}\right)$ as repeated measure factor. Subject was nested in group and included as a random effect in all analyses. The selected covariance structure was first-order ante dependence for the analysis of cortisol reactivity, and autoregressive(1) for the analysis of basal cortisol levels. Since the residuals of the calculated models were not normally distributed, we repeated all our analyses with a logarithmized data set. In the case of significant effects we computed individual ANOVAs for each group. Furthermore, $t$-tests for independent samples were calculated to test for group differences at the separate time points. Applying Akaikes information criterion (AIC) for model selection, covariates were included in our model, if their introduction yielded a better model fit compared to the original model and reduced $\mathrm{AIC}$ at least by 2 points (Burnham and Anderson, 2002, 2004).

Correlations between cortisol basal levels $\left(t_{1}\right.$ and $\left.t_{4}\right)$ as well as correlations between salivary and plasma cortisol levels were computed using the nonparametric correlation coefficient Kendalls $\tau$.

\section{Results}

\subsection{Clinical characteristics}

Trauma groups (raped vs. non-raped) did not significantly differ with respect to age, educational level, number of different traumatic event types, PTSD symptom severity, the mean duration of PTSD symptoms and the severity of depressive symptoms (Table 1). However, subjects who had been raped tended to report more somatic symptoms.

Moreover, groups did not significantly differ with respect to the duration of the individual sections of the interview [from $t_{1}$ to start of trauma confrontation, $t(23)=-.42$, $p=.67$; from start of trauma confrontation to $t_{2}$, $t(21)=-.80, p=.43$; from $t_{2}$ to $\left.t_{3}, t(19)=-.63, p=.54\right]$.

\subsection{Cortisol reactivity}

Salivary cortisol. The analysis of salivary cortisol levels showed a significant main effect of time $[F(2,29.5)=5.39$, $p \leq .01]$, no main effect of trauma type $[F(1,28.4)=.94$, $p=.34]$ and a significant time $\times$ trauma type interaction $[F(2,29.5)=12.67, p<.001]$. As displayed in Fig. 2A, trauma groups did not significantly differ in basal $\left[t_{1}, t(25.7)=1.18\right.$, $p=.25]$ or post-stressor $\left[t_{3}, t(27)=-.79, p=.43\right]$ salivary cortisol levels. However, differential cortisol responses were observed in PTSD patients who had been raped compared to those who had not been raped [ $\left.t_{2}, t(27)=-2.35, p<.05\right]$. Individual ANOVAs computed for each trauma group revealed significant main effects of time for both trauma groups [raped: $\quad F(2,9.9)=17.97, \quad p<.001 ; \quad$ non-raped: $F(2,22.6)=8.65, p=.002]$, indicating that cortisol levels decreased in the course of the interview for the group with no past experience of rape ( $t_{1}$ vs. $t_{2}$ and $t_{2}$ vs. $t_{3}, p<.05$ ). In contrast, PTSD patients who had been raped showed a significant cortisol increase when reminded of their traumatic events ( $t_{1}$ vs. $\left.t_{2}, p<.001\right)$, and returned to baseline levels at $t_{3}\left(t_{2}\right.$ vs. $t_{3}, p=.11 ; t_{1}$ vs. $\left.t_{3}, p=.92\right)$. When repeating these analyses after log-transformation of the cortisol data, the result pattern did not change.

Since our sample consisted of male and female participants as well as of individuals with and without a comorbid

Table 1 Demographic and clinical characteristics of raped and non-raped PTSD patients.

\begin{tabular}{|c|c|c|c|c|c|c|}
\hline \multirow[t]{2}{*}{ Variables } & \multicolumn{2}{|l|}{ Raped } & \multicolumn{2}{|c|}{ Non-raped } & \multirow[t]{2}{*}{$t(28)$} & \multirow[t]{2}{*}{$p$ value } \\
\hline & M & SD & $M$ & SD & & \\
\hline Age & 34.00 & 10.2 & 33.7 & 8.7 & -.07 & .94 \\
\hline Educational level (years in school) & 7.45 & 2.98 & 8.25 & 3.75 & -.58 & .56 \\
\hline Number of CAPS events & 7.40 & 2.17 & 6.55 & 2.01 & -1.06 & .29 \\
\hline Number of war and torture event types & 11.90 & 6.31 & 10.10 & 5.33 & -.82 & .42 \\
\hline CAPS score & 78.10 & 17.92 & 78.95 & 17.82 & .12 & .90 \\
\hline Duration of PTSD symptoms (in years) & 6.69 & 3.78 & 7.16 & 5.19 & -.25 & .80 \\
\hline HAM-D score & 27.80 & 7.67 & 24.90 & 8.52 & -.91 & .37 \\
\hline SOMS-7 score & 36.40 & 10.85 & 27.84 & 12.95 & -1.78 & .08 \\
\hline
\end{tabular}

CAPS, Clinician Administered PTSD Scale; HAM-D, Hamilton Depression Rating Scale; SOMS-7, Screening for Somatoform Symptoms-7. 

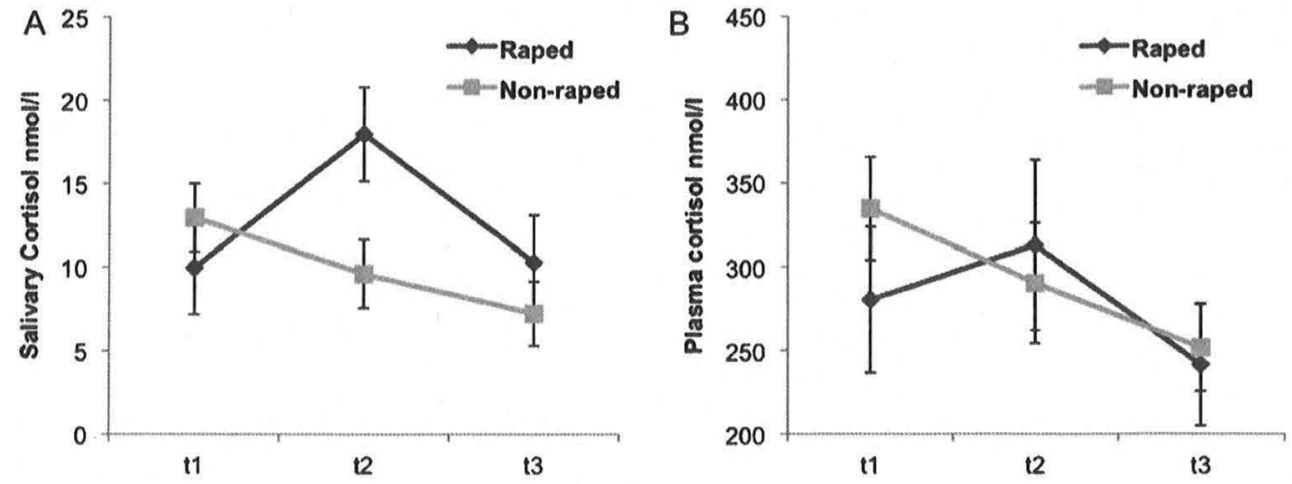

Figure 2 Salivary (A) and plasma (B) cortisol responses in raped and non-raped PTSD patients. Patients underwent a standardized clinical interview and samples were collected at the beginning of the interview $\left(t_{1}\right)$, directly after the assessment of traumatic experiences $\left(t_{2}\right)$, and after completion of the Clinician-Administered PTSD Scale $\left(t_{3}\right)$. Data are presented as mean (LSM) \pm SE.

depressive episode, we repeated our analyses with gender or current depression as additional fixed effects. For salivary cortisol, no significant main effect of gender $[F(1,26.5)=.22, p=.88]$, no significant time $\times$ gender interaction $[F(2,26.7)=1.46, p=.25]$, and no significant time $x$ trauma type $\times$ gender interaction $[F(2,26.7)=1.11$, $p=.34]$ could be identified. Moreover, no significant main effect of current depression $[F(1,27.0)=2.56, p=.12]$, and no significant time $\times$ current depression interaction $[F(2,27.7)=1.32, p=.28]$ could be detected. After introducing gender or current depression as additional fixed effects, all major effects reported above remained statistically significant.

Plasma cortisol. For total plasma cortisol, we found a significant main effect of time $[F(2,32.1)=4.01, p<.05]$. Cortisol levels at $t_{2}$ were not different from baseline ( $t_{1}$ vs. $\left.t_{2}, p=.78\right)$, but declined significantly in the course of the interview ( $t_{1}$ vs. $t_{3}$, and $t_{2}$ vs. $\left.t_{3}, p<.05\right)$. There was no significant main effect of trauma type $[F(1,28.4)=.86$, $p=.77]$, or time $\times$ trauma type interaction $[F(2,32.1)=1.67, p=.20]$, see Fig. 2B. For the logarithmized plasma cortisol data we found a significant time $\times$ trauma type interaction $[F(2,33.5)=3.82, p<.05]$. However $t$-tests failed to show significant group differences at $t_{1}, t_{2}$ and $t_{3}$. Individual ANOVAs computed for each trauma group revealed a significant main effect of time for the non-raped group $[F(2,26.2)=5.22, p<.05]$, showing that cortisol levels decreased in the course of the interview ( $t_{1}$ vs. $t_{2}$ and $t_{1}$ vs. $\left.t_{3}, p<.05\right)$. In contrast, no significant main of time could be detected for the raped group $[F(2,9.7)=3.3, p=.08]$.

When repeating these analyses with gender or current depression as additional fixed effects, the ANOVA showed no significant main effect of gender $[F(1,26.5)=.17, p=.68]$, no significant time $\times$ gender interaction $[F(2,29.6)=1.41$, $p=.26]$, and no significant time $\times$ trauma type $\times$ gender interaction $[F(2,29.6)=.13, p=.88]$. After introducing gender as additional fixed effect, all major effects reported above remained statistically significant. In contrast, we found a significant main effect of current depression $[F(1,28.1)=7.61, p=.01]$, indicating that depressed individuals had significantly higher overall plasma cortisol levels, but no significant time $\times$ current depression interaction $[F(2,30.1)=1.63, p=.21]$. Moreover, after introducing current depression as additional fixed effect, the main effect of time reported above remained no longer statistically significant.
In a further step, we introduced the variables smoking, or awakening time, or time since the worst event occurred as covariates to the original model. This changed only the main effect of time, which was no longer significant, but did not significantly alter the other results. For none of these variables we could identify a significant main effect, a significant interaction of time $\times$ covariate, or a significant interaction of time $\times$ trauma type $\times$ covariate.

\subsection{Basal cortisol measurement}

We further compared basal ( $t_{1}$ and $\left.t_{4}\right)$ salivary and plasma cortisol secretion of the combined PTSD patient group with those of the non-PTSD control group. With respect to salivary cortisol, we found no significant main effect of time $[F(1,51.5)=.63, p=.43]$, indicating that cortisol levels did not differ between the two basal measurement points, see Fig. 3A. Furthermore, there was no significant main effect of diagnosis $[F(1,54.1)=.07, p=.79]$, and no significant time $x$ diagnosis interaction $[F(1,51.5)=3.14, p=.08]$. Nonparametric correlation analyses revealed that basal salivary probes $\left(t_{1}\right.$ and $\left.t_{4}\right)$ were significantly related in the control (Kendalls $\tau=.56, p<.001$ ) as well as the PTSD group (Kendalls $\tau=.53, p<.001$ ).

For total plasma cortisol levels, again no main effect of time $[F(1,54.0)=.11, p=.74]$, and no significant time $\times$ diagnosis interaction $[F(1,54.0)=.78, p=.38]$ was detected. However, as depicted in Fig. 3B, there was a trend for a main effect of diagnosis $[F(1,55.9)=3.72, p=.06]$, indicating that individuals with PTSD had lower total plasma concentrations than the non-PTSD control group. In both groups plasma cortisol basal levels $\left(t_{1}\right.$ and $\left.t_{4}\right)$ were significantly related (controls: Kendalls $\tau=.59, p<.001$; PTSD: Kendalls $\tau=.42, p \leq .001$ ).

When repeating these analyses after log-transformation of the cortisol data, results did not significantly change.

\subsection{Correlation of salivary and plasma cortisol levels}

Nonparametric correlation analyses revealed significant small to moderate size associations between salivary and plasma cortisol measures for the whole PTSD patients group 

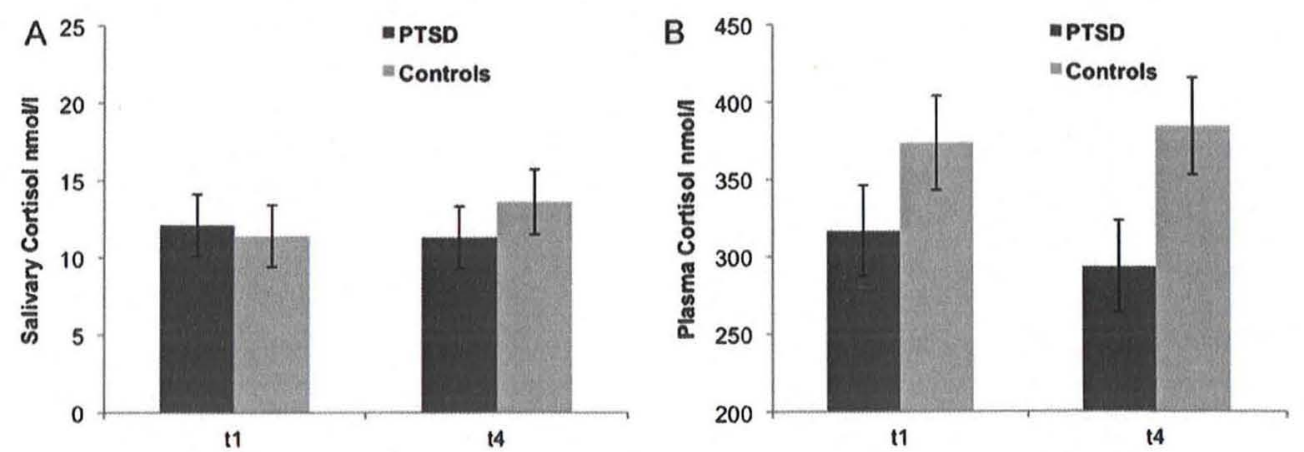

Figure 3 Basal salivary (A) and plasma (B) cortisol levels in the combined PTSD patient group and the control group. Samples were collected at the beginning of the clinical interview $\left(t_{1}\right)$ as well as one week afterwards at the same time of the day $\left(t_{4}\right)$ to control for the stability of basal cortisol excretion. Data are presented as mean (LSM) $\pm \mathrm{SE}$.

at $t_{1}$ (Kendalls $\left.\tau=.32, p<.05\right), t_{2}$ (Kendalls $\tau=.35, p<.01$ ) and $t_{3}$ (Kendalls $\tau=.30, p<.05$ ) as well as for the control group at $t_{1}$ (Kendalls $\tau=.31, p<.05$ ).

\section{Discussion}

This study investigated the effect of trauma type (rape vs. other trauma types) on salivary and plasma cortisol responses of PTSD patients after being confronted with trauma reminders. Trauma groups had comparable basal salivary or plasma cortisol levels, however, our results demonstrate differential salivary cortisol responses in PTSD patients who had been raped compared to those who had not been raped, but who had experienced an equal number of severe traumatic incidences and showed equally high PTSD symptom severity. Consistent with a typical diurnal decline, salivary cortisol levels decreased in the course of the interview for the group with no past experience of rape, whereas those PTSD patients who had been raped showed a significant salivary cortisol increase when reminded of their traumatic events. This effect remained also significant, when gender or comorbid depression was included in the analysis. In contrast, we did not find an effect for trauma type on plasma cortisol responses. To control for possible anticipatory anxiety effects regarding the procedure, as reported in some previous studies (Bremner et al., 2003; Elzinga et al., 2003), we further compared basal cortisol levels of the PTSD patients group with cortisol levels of a healthy control group on two different days. Whereas salivary cortisol levels of PTSD patients were not significantly different from a group of healthy controls, PTSD patients tended to show lower plasma basal cortisol levels, a result that is in line with findings of a number of previous studies (Yehuda, 2001) and argues against elevated basal cortisol levels induced by anticipatory anxiety.

Results indicate that salivary cortisol responses during the confrontation with trauma-related material are affected by the particular set of traumatic stressors a patient has experienced. This is in accordance with studies that found a pronounced cortisol response to trauma reminders following sexual trauma (Elzinga et al., 2003), but no such responses with respect to other trauma types (Liberzon et al., 1999; Kolassa et al., 2007). The nearness of the perpetrator during the traumatic event might modulate peritraumatic physiologic responses, as well as later cortisol responses to trauma reminders. The direct physical contact with a perpetrator during sexual assault combined with extreme fear, could predispose sexual assault victims to display tonic (Suarez and Gallup, 1979; Marx et al., 2008) or flaccid (Schauer and Elbert, 2010) immobility, i.e. a shutdown response, when flight or fight has proven unsuccessful. This last survival strategy should be especially likely when bodily contact becomes maximal, as is the case when the sexual assault includes the act of vaginal or anal coitus, as reported by the subjects in our study. This interpretation is supported by the anecdotal report of "shutdown behavioral patterns" within the raped group, observed by the clinicians conducting the interviews during the assessment of traumatic experiences (e.g., subjects did not hear, see or perceive well anymore, they moved less, and had a hard time to understand language and were unwilling to produce speech). Moreover, in animal studies it has been found that a behavioral style to counteract a threatening situation that includes active avoidance and aggression, is associated with high sympathetic reactivity and low glucocorticoid secretion, while passive coping strategies such as immobility and withdrawal are associated with low sympathetic reactivity and high glucocorticoid release (Korte et al., 2005). Furthermore, experimentally induced immobility responses in animals were associated with increased release of hypothalamus-pituitary-adrenal (HPA) axis hormones (Farabollini et al., 1990).

However, since rape victims might differ from other trauma victims not only in their peritraumatic bodily reactions but also in their emotional responses towards the trauma, there are other possible explanations to consider when discussing the present findings. For example, it has been reported that rape victims display high amounts of shame during and following their assault (Foa and Rothbaum, 1998; Kaysen et al., 2005). In studies in which healthy subjects are exposed to social evaluation in a laboratory setting, the extent of cortisol reactivity increases in a linear fashion with shame (Dickerson and Kemeny, 2004; Gruenewald et al., 2004), suggesting that feelings of shame foster HPA activation during acute stress periods (Miller et al., 2007). Thus, feelings of shame in the presence of the interviewer during the admittance of sexual assault during the diagnostic interview or feelings of shame re-triggered by trauma reminders might constitute an additional relevant explanation for our results. However, in our sample poten- 
tially shameful experiences were not only reported by rape victims but also from PTSD patients from the non-raped group (e.g., unwanted touching, twisting or squeezing of genitals during torture, witnessing family members being sexually assaulted), who did not show salivary cortisol reactions during the report of these events. Moreover, in PTSD patients, shame seems to be positively correlated with PTSD symptom severity (Leskela et al., 2002), and our two trauma groups did not differ with respect to the severity of their symptoms.

In contrast to the pronounced effect of trauma type on salivary cortisol levels which primarily reflects the unbound, active hormone portion, we did not find an effect for trauma type on total plasma cortisol composed of active and bound hormone fractions. This could be due to a reduction of the binding capacity of cortisol binding globulins (CBG) in response to the trauma-associated stressor and a resulting increase in the proportion of unbound, bioavailable cortisol, which has been documented in response to stress by several studies (Spencer et al., 1996; Alexander and Irvine, 1998; Engler et al., 2004). Alternatively, the tendency of lower basal plasma cortisol values within the raped group (see Fig. 2B) might have precluded a significant time $\times$ trauma type interaction in plasma cortisol. This tendency is in accordance with the results of the meta-analysis of Meewisse et al. (2007), showing significantly lower basal blood cortisol levels in people with PTSD due to sexual abuse than in controls, whereas no differences in cortisol level were found between controls and people with PTSD due to other types of trauma - thus suggesting that victims of sexual violence show lower basal cortisol values in plasma or serum. The varying basal plasma cortisol values in the two trauma groups might be caused by differing concentrations or binding capacities of CGB for raped PTSD patients compared to PTSD patients with other trauma types - an issue that should be addressed in future studies.

In sum, our results support the idea that different physiological responses of the body when being reminded of a trauma exist depending on the type of traumatic stress experienced. Important strengths of the study include the measurement of two groups of severely traumatized PTSD patients that differed only in the type of traumatic events experienced (rape, other traumatic events) but were comparable with respect to traumatic load and severity of PTSD symptoms. Moreover, we could preclude that gender accounts for the observed effects, since our sample consisted not only of women, but also of men with a history of rape. Factors limiting the interpretation of our data comprise (1) the lack of a healthy control group with respect to cortisol reactivity to trauma reminders, (2) the variation in the completion times of sections interviews, (3) the intake of psychotropic medication in $1 / 3$ of the PTSD patients, which was, however, at least equally distributed across groups and thus should not be the factor accounting for the observed effects, (4) the diverse ethnicities of the subjects, and (5) the relatively small sample size. Since the experience of immobility is not necessarily bound to rape, but may also be displayed during other trauma types associated with fear, contact and restraint (e.g. certain forms of torture), future studies on cortisol reactivity should include assessments of peritraumatic responding in addition to comparisons of trauma type.

\section{Role of the funding source}

This study was funded by the German Research Foundation (DFG) FOR751 and the European Refugee Fund. The DFG and the European Refugee Fund had no further role in study design; in the collection, analysis and interpretation of data; in the writing of the report; and in the decision to submit the paper for publication.

\section{Conflict of interest}

None declared.

\section{Acknowledgments}

We thank Claudia Catani, Frank Neuner and Martina Ruf for clinical supervision and treatment of patients, and Heike Riedke and Christiane Wolf for technical assistance.

\section{References}

Alexander, S.L., Irvine, C.H., 1998. The effect of social stress on adrenal axis activity in horses: the importance of monitoring corticosteroid-binding globulin capacity. J. Endocrinol. 157, $425-432$.

American Psychiatric Association, 1994. Diagnostic and Statistical Manual of Mental Disorders (DSM-IV). American Psychiatric Association, Washington, DC.

Blake, D.D., Weathers, F.W., Nagy, L.M., Kaloupek, D.G., Gusman, F.D., Charney, D.S., Keane, T.M., 1995. The development of a Clinician-Administered PTSD Scale. J. Trauma. Stress 8, 75-90.

Bremner, J.D., Vythilingam, M., Vermetten, E., Adil, J., Khan, S., Nazeer, A., Afzal, N., McGlashan, T., Elzinga, B., Anderson, G.M., Heninger, G., Southwick, S.M., Charney, D.S., 2003. Cortisol response to a cognitive stress challenge in posttraumatic stress disorder (PTSD) related to childhood abuse. Psychoneuroendocrinology 28, 733-750.

Burgess, A.W., Holmstrom, L.L., 1976. Coping behavior of the rape victim. Am. J. Psychiatry 133, 413-418.

Burnham, K.P., Anderson, D.R., 2002. Model Selection and MultiModel Inference: A Practical Information-Theoretic Approach. Springer.

Burnham, K.P., Anderson, D.R., 2004. Multimodel inference: understanding AIC and BIC in model selection. Sociol. Methods Res. 33, $261-304$.

de Kloet, C.S., Vermetten, E., Geuze, E., Kavelaars, A., Heijnen, C.J., Westenberg, H.G., 2006. Assessment of HPA-axis function in posttraumatic stress disorder: pharmacological and non-pharmacological challenge tests, a review. J. Psychiatr. Res. 40, $550-567$.

Dickerson, S.S., Kemeny, M.E., 2004. Acute stressors and cortisol responses: a theoretical integration and synthesis of laboratory research. Psychol. Bull. 130, 355-391.

Elzinga, B.M., Schmahl, C.G., Vermetten, E., van Dyck, R., Bremner, J.D., 2003. Higher cortisol levels following exposure to traumatic reminders in abuse-related PTSD. Neuropsychopharmacology 28, 1656-1665.

Engler, H., Dawils, L., Hoves, S., Kurth, S., Stevenson, J.R., Schauenstein, K., Stefanski, V., 2004. Effects of social stress on blood leukocyte distribution: the role of alpha- and beta-adrenergic mechanisms. J. Neuroimmunol. 156, 153-162.

Farabollini, F., Facchinetti, F., Lupo, C., Carli, G., 1990. Time-course of opioid and pituitary-adrenal hormone modifications during the immobility reaction in rabbits. Physiol. Behav. 47, 337-341. 
Foa, E.B., Kozak, M.J., 1986. Emotional processing of fear: exposure to corrective information. Psychol. Bull. 99, 20-35.

Foa, E.B., Rothbaum, B.O., 1998. Treating the Trauma of Rape: Cognitive-Behavioral Therapy for PTSD. The Guilford Press, New York.

Fuse, T., Forsyth, J.P., Marx, B., Gallup, G.G., Weaver, S., 2007. Factor structure of the Tonic Immobility Scale in female sexual assault survivors: an exploratory and Confirmatory Factor Analysis. J. Anxiety Disord. 21, 265-283.

Galliano, G., Noble, L., Travis, L.A., Puechl, C., 1993. Victim reactions during rape/sexual assault: a preliminary study of the immobility response and its correlates. J. Interpers. Violence 8, 109-114.

Griffin, M.G., Resick, P.A., Mechanic, M.B., 1997. Objective assessment of peritraumatic dissociation: psychophysiological indicators. Am. J. Psychiatry 154, 1081-1088.

Gruenewald, T.L., Kemeny, M.E., Aziz, N., Fahey, J.L., 2004. Acute threat to the social self: shame, social self-esteem, and cortisol activity. Psychosom. Med. 66, 915-924.

Hamilton, M., 1960. A rating scale for depression. J. Neurol. Neurosurg. Psychiatry 23, 56-62.

Heidt, J.M., Marx, B.P., Forsyth, J.P., 2005. Tonic immobility and childhood sexual abuse: a preliminary report evaluating the sequela of rape-induced paralysis. Behav. Res. Ther. 43, 1157-1171.

Kaysen, D., Morris, M.K., Rizvi, S.L., Resick, P.A., 2005. Peritraumatic responses and their relationship to perceptions of threat in female crime victims. Violence Against Women 11, 1515-1535.

Keane, T.M., Zimering, R.T., Caddell, J.M., 1985. A behavioral formulation of posttraumatic stress disorder in Vietnam veterans. Behav. Ther. 8, 9-12.

Kolassa, I.T., Eckart, C., Ruf, M., Neuner, F., de Quervain, D.J., Elbert, T., 2007. Lack of cortisol response in patients with posttraumatic stress disorder (PTSD) undergoing a diagnostic interview. BMC Psychiatry 7, 54.

Korte, S.M., Koolhaas, J.M., Wingfield, J.C., McEwen, B.S., 2005. The Darwinian concept of stress: benefits of allostasis and costs of allostatic load and the trade-offs in health and disease. Neurosci. Biobehav. Rev. 29, 3-38.

Lang, P.J., 1979. A bio-informational theory of emotional imagery. Psychophysiology 16 , 495-512.

Lanius, R.A., Bluhm, R., Lanius, U., Pain, C., 2006. A review of neuroimaging studies in PTSD: heterogeneity of response to symptom provocation. J. Psychiatr. Res. 40, 709-729.

Lanius, R.A., Vermetten, E., Loewenstein, R.J., Brand, B., Schmahl, C., Bremner, J.D., Spiegel, D., 2010. Emotion modulation in PTSD: clinical and neurobiological evidence for a dissociative subtype. Am. J. Psychiatry 167, 640-647.

Lanius, R.A., Williamson, P.C., Boksman, K., Densmore, M., Gupta, M., Neufeld, R.W., Gati, J.S., Menon, R.S., 2002. Brain activation during script-driven imagery induced dissociative responses in PTSD: a functional magnetic resonance imaging investigation. Biol. Psychiatry 52, 305-311.

Leskela, J., Dieperink, M., Thuras, P., 2002. Shame and posttraumatic stress disorder. J. Trauma. Stress 15, 223-226.
Liberzon, I., Abelson, J.L., Flagel, S.B., Raz, J., Young, E.A., 1999. Neuroendocrine and psychophysiologic responses in PTSD: a symptom provocation study. Neuropsychopharmacology 21, 40-50.

Lindauer, R.J., Olff, M., van Meijel, E.P., Carlier, I.V., Gersons, B.P., 2006. Cortisol, learning, memory, and attention in relation to smaller hippocampal volume in police officers with posttraumatic stress disorder. Biol. Psychiatry 59, 171-177.

Marx, B.P., Forsyth, J.P., Gallup, G.G., Fusé, T., Lexington, J.M., 2008. Tonic immobility as an evolved predator defense: implications for sexual assault survivors. Clin. Psychol. Sci. Pract. 15, 74-90.

Meewisse, M.L., Reitsma, J.B., de Vries, G.J., Gersons, B.P., Olff, M., 2007. Cortisol and post-traumatic stress disorder in adults: systematic review and meta-analysis. Br. J. Psychiatry 191, 387 392.

Miller, G.E., Chen, E., Zhou, E.S., 2007. If it goes up, must it come down? Chronic stress and the hypothalamic-pituitary-adrenocortical axis in humans. Psychol. Bull. 133, 25-45.

Orr, S.P., Roth, W.T., 2000. Psychophysiological assessment: clinical applications for PTSD. J. Affect. Disord. 61, 225-240.

Pole, N., 2007. The psychophysiology of posttraumatic stress disorder: a meta-analysis. Psychol. Bull. 133, 725-746.

Rief, W., Hiller, W., 2003. A new approach to the assessment of the treatment effects of somatoform disorders. Psychosomatics 44, 492-498.

Schauer, M., Elbert, T., 2010. Dissociation following traumatic stress: etiology and treatment. Z. Psychol. 218, 109-127.

Schauer, M., Neuner, F., Elbert, T., 2011. Narrative Exposure Therapy, 2nd ed. Hogrefe \& Huber, Göttingen, Germany.

Sheehan, D.V., Lecrubier, Y., Sheehan, K.H., Amorim, P., Janavs, J. Weiller, E., Hergueta, T., Baker, R., Dunbar, G.C., 1998. The MiniInternational Neuropsychiatric Interview (M.I.N.I.): the development and validation of a structured diagnostic psychiatric interview for DSM-IV and ICD-10. J. Clin. Psychiatry 59 (Suppl 20), 22-33.

Spencer, R.L., Miller, A.H., Moday, H., McEwen, B.S., Blanchard, R.J., Blanchard, D.C., Sakai, R.R., 1996. Chronic social stress produces reductions in available splenic type II corticosteroid receptor binding and plasma corticosteroid binding globulin levels. Psychoneuroendocrinology 21, 95-109.

Suarez, S.D., Gallup, G.G., 1979. Tonic immobility as a response to rape in humans: a theoretical note. Psychol. Rec. 29, 315-320.

Tucker, P., Ruwe, W.D., Masters, B., Parker, D.E., Hossain, A., Trautman, R.P., Wyatt, D.B., 2004. Neuroimmune and cortisol changes in selective serotonin reuptake inhibitor and placebo treatment of chronic posttraumatic stress disorder. Biol. Psychiatry 56, 121-128.

Wessa, M., Rohleder, N., Kirschbaum, C., Flor, H., 2006. Altered cortisol awakening response in posttraumatic stress disorder. Psychoneuroendocrinology 31, 209-215.

Yehuda, R., 2001. Biology of posttraumatic stress disorder. J. Clin. Psychiatry 62 (Suppl. 17), 41-46.

Yehuda, R., Kahana, B., Binder-Brynes, K., Southwick, S.M., Mason, J.W., Giller, E.L., 1995. Low urinary cortisol excretion in Holocaust survivors with posttraumatic stress disorder. Am. J. Psychiatry $152,982-986$. 